\title{
Yoga and Depressive Disorders: A Review of Current Literature
}

\author{
Jennifer Hofmann* \\ Department of Physical Therapy, USA
}

*Corresponding author: Jennifer Hofmann, Department of Physical Therapy, USA.

Received Date: September 30, 2019

Published Date: October 16, 2019

\section{Introduction}

Depressive disorders are among the most common mental health disorders encountered in primary care. The most common initial treatment for major depressive disorder includes a combination of pharmacotherapy and psychotherapy. Although these modalities are effective adherence issues are common; and complementary and alternative approaches may provide additional options for depressive disorders. Yoga is the most commonly used complementary health approach in the United States of America (USA) [1]. There is some clinical evidence that supports the benefits of yoga for depressive disorders, but the results are variable. The article reviews data from current randomized clinical trials, systematic reviews and a meta-analysis on the effectiveness of yoga for depressive disorders (Appendix).

\section{Research Design and Review of Evidence}

Prathikanti S, et al. [1] conducted an 8week prospective, singlecenter, single-blind, randomized, controlled, parallel group pilot trial to investigate the benefits of hatha yoga as monotherapy for mild-to-moderate major depression2. Participants were assessed by the PI, a physician, and were included if they were: English speaking adults with mild to moderate major depression not currently taking antidepressants including herbal supplements, not practicing yoga and not in psychotherapy.

Participants were randomly assigned to either active or control arms via computer and assignments were concealed from the PI. 38 participants were randomized to either the active hatha yoga practice sessions arm $(n=20)$ or the attention control group/ yoga education modules $(n=18)$. Baseline characteristics were similar in both groups. Primary outcome measure was depression severity, measured by BDI scores at baseline and in 2 week increments throughout the study. The analysis of primary outcome used an intent to treat approach and included data from all randomized participants.

The results demonstrate a statistically significant change in depression severity (primary outcome) on the BDI from baseline to the final 8 week assessment. There was also a greater decline of depression severity over the 8week period in the yoga group compared to the control group. The authors also estimated the effect size of yoga on primary and secondary outcomes as relatively large. This study had some limitations including a small sample size which limits study power and short duration of intervention.

Uebelacker L, et al. [3] conducted a 10 week randomized controlled trial to determine the effectiveness of hatha yoga as an adjunctive therapy to antidepressants in patients with major depression3. Essentially participants were eligible if: they met the criteria for major depression via the Structured Clinical Interview for DSM-IV(SCID), were currently taking effective doses of antidepressants, in stable psychotherapy and with minimal exposure to yoga practice3.122 participants were randomized using a computer program into 2 groups; 104 participants completed the intervention phase. The 2 groups had similar baseline characteristics. The active intervention arm was yoga (80 minutes per session 1-2 times per week for 10 weeks). The control arm included concurrent healthy living workshop classes for 60 minutes 1-2 times per week. The primary outcome was depression severity assessed at several points during the study and at 3 and 6 month follow ups. The interviewers who assessed participants during the study were blinded to the intervention assignments. At week 10, there was no statistically significant difference in the primary outcome between groups. However, when depression severity scores were compared 
over the entire intervention and at follow up periods, the yoga participants demonstrated significantly lower depression severity. Secondary outcomes such as self rated depressive symptoms and overall functioning were also better in the yoga arm. This RCT demonstrated that in a largely white female population with persistent depression yoga did not seem to decrease depressive symptoms acutely but did improve depressive severity and other outcomes at 3 and 6 month follow ups.

Cramer $\mathrm{H}$, et al. [4] present a systematic review and metaanalysis to evaluate the effectiveness of yoga for depression. 4 Nine RCTs with 452 participants were included and 3 of the 9 RCTs had low risk of bias, which was assessed using the using the risk of bias tool proposed by the Cochrane Back Review Group . Patient selection criteria included adults with primary depressive disorders and/ or elevated levels of depression. The intervention assessed included yoga of different types (exercise, complex or meditative). Yoga intervention was compared to heterogenous controls including no treatment, pharmacotherapy, hypnosis, relaxation, exercise, music, massage and social groups. The duration of studies varied with a median of 8 weeks. Outcome measures included severity of depression in all studies (measured by validated scales and one unvalidated scale). Some studies also reported outcomes of remission rates and severity of anxiety. The meta-analysis concluded that there is limited to moderate evidence that yoga, specifically meditation or breathing based yoga produced a shortterm reduction in the severity of depression and improvement in elevated levels of depression. In this meta analysis the effects of yoga were similar to other interventions including massage, antidepressant medication, social support or group therapy.

There were some significant limitations of the RCTs in this meta-analysis. The methodology of most of the included RCTs was poor with lack of blinding, heterogeneous experimental interventions e.g. variable types and intensity of yoga practice and variable control intervention groups and inadequate allocation concealment. There was high attrition in most of the RCTS and only 2 trials reported longer term effects; none of the RCTs assessed safety measures.

Although this meta-analysis suggests that yoga may improve the severity of depression, larger, well designed and longer term RCTs will further clarify the role of yoga intervention in patients with depressive disorders. In this article, De Manincor, et al. [5]. conducted a RCT to evaluate the effectiveness of 6 weeks of yoga intervention in the reduction of symptoms of depression.5 101 eligible participants were randomized to either; 6-week yoga plus treatment-as-usual (TAU) intervention group, or a waitlist plus TAU control group.5 The TAU control group was offered yoga at the end of 6 weeks of the active trial. Basic inclusion criteria included: adults with stable TAU (medications and/or professional mental assistance), and Depression, Anxiety and Stress Scale DASS21 scores consistent with at least mild depression. Patients were excluded if they were currently practicing yoga regularly, drug or alcohol frequent users and/or had serious medical disorders.
Participants were blinded to group allocations and yoga instructors were blinded to initial group allocations. The yoga intervention included: (4) onehour teaching /consultation sessions conducted over 6 weeks with individualized guides for at home practice. The yoga practice included physical postures, breathing, relaxation, mindfulness and meditation. The details of each participants' at home practice were recorded at each session.

Results of the trial: The DASS-21 was used as the primary outcome measure to assess depression and anxiety. Kessler Psychological Distress Scale and the Short-Form 12-Item Health Survey were used as secondary outcome measures for depression, anxiety and psychological distress. There was statistically significant reduction in DASS depression scores in the yoga group compared to the waitlist control group. The yoga group had an increased frequency in the reduction of their usual therapies compared to the control group. There were statistically significant differences among groups for most secondary outcome measures except for stress scores and physical health. The benefits of yoga continued in a 6 week follow-up assessment. Yoga was clinically significant in reducing symptoms of depression compared to control TAU.

This study demonstrated the effectiveness of yoga intervention with regular classic yoga practice for reducing symptoms of depression. It also demonstrated positive effects on mental wellbeing and reduction in anxiety symptoms with no adverse effects. The benefits continued in the 6week post-study follow up in participants continuing their yoga practice.

There were some notable limitations to the trial. Examiners used a single assessment used at the screening session. This trial included a volunteer sample of people who were open to yoga practice which may limit general applicability. Many participants had both depression and comorbid anxiety and there was a small number of patients with exclusively depression; this makes it difficult to clarify the effects of yoga for each disorder. The effects of yoga were only compared to TAU but not to another active intervention such as exercise. Participants self-reported measures of symptoms to teachers and other data regarding effectiveness such as clinician evaluations were not assessed.

In this article Falsafi $N$ [6]. conducted a RCT in college students with depression and/or anxiety to compare the effects of mindfulness versus yoga interventions on the severity of depression.6 90 college students with a diagnosis of either depression or anxiety were randomized to one of three groups: yoga intervention, mindfulness intervention or non intervention control group for 12 weeks. Participants in the intervention groups received 8 weeks (75 minutes per week) of either mindfulness training and self compassion or Hatha yoga instruction (75 minutes/ week) with instructions for at home practice. They completed validated symptom questionnaires the Beck Depression Inventory and the Hamilton Anxiety scale before training, mid training, at the end of the last session (8weeks) and 4 weeks after study completion. 
Participants were given a journal to document daily home practice and any significant changes in treatment or life events.

The sessions were conducted by the same psychiatric clinical nurse specialist who was a certified holistic nurse and certified yoga teacher. 67 students completed the entire study which represented a 25\% attrition rate. Many of the participants had comorbid depression and anxiety and only 7 had depression only. The majority were taking psychiatric medications and 15 students were receiving therapy during the study. Results of the trial demonstrated that the symptoms of depression decreased significantly in both the yoga $(p<0.01)$ and mindfulness intervention group $(p<0.01)$ compared to the control group from pre to follow up measurements. 4 There were non-significant changes in the control group. In addition to data collected from the questionnaires, qualitative data from daily journals and a summary of the important events was completed at the last day of the study.

This study provides further evidence supporting the effectiveness of yoga intervention to improve mood and decrease symptoms of depression. The study used a control group and another intervention group, mindfulness practice, which was also effective for depressed participants. Each of these interventions were effective; this provides options for offering one or both depending on patients preferences. Based on the results from this trial and evidence from similar trials clinicians may consider recommending yoga practice as an adjunctive therapy for depressive disorders.

Limitations of this study include a small and relatively homogenous population of young mostly Causacian female participants. The participants self reported symptoms of depression with questionnaires before the training sessions, mid study, on the last day and 4 weeks after the completion which may limit accuracy or full account of effects. There was no mention of blinding of any study participants. The study did not explicitly assess if there was a significant difference between the yoga intervention and mindfulness intervention effects on depression.

Cramer $\mathrm{H}$, et al. [7] present a systematic review to assess the effectiveness and safety of yoga interventions in treating patients with major depressive disorder. 7 The purpose of this clinical review article focuses on the effectiveness of yoga for depression. 7 RCT with 240 participants were included in this review; all 7 studies enrolled participants who met DSM IV criteria for depressive disorders. The interventions in each trial were either yoga with physical postures, breathing and meditation or yoga without postures for a median duration of 8 weeks, with a median of 2 sessions per week. 2 RCT used standard care with antidepressants as the control group and the other 5 trials used variable active control groups including medication, walking therapy and electroconvulsive therapy.

The main outcome measure was the effect of yoga compared to control (standard care or active controls) on the severity of depression. Outcomes were assessed at the end of the intervention for each trial. The trials used instruments such as the Hamilton
Rating Scale for Depression, Quick Inventory of Depressive Symptoms and the Beck Depression Inventory to assess depressive symptoms.

Yoga interventions provided similar statistically significant reductions in the severity of depressive symptoms when compared to exercise (home-walking program) and/or imipramine antidepressant medication. Electroconvulsive therapy was more effective than yoga for treating depression in a 4week randomized open clinical trial. One trial demonstrated that yoga added to antidepressants significantly improved depressive and anxious symptoms compared to the medication control group. By contrast in a RCT Hatha yoga was added to medication as intervention group compared to medication alone as control; this study found no significant difference between the two groups.

There were some limitations of this systematic review. Each of the studies analyzed had a small sample size. The interventions were heterogeneous as were the control groups; lack of appropriate methodology such as blinding, appropriate sample size, intention to treat analysis and adequate randomization limited applicability of trial results [7].

\section{Conclusion}

Overall, these studies support the effectiveness of yoga compared to usual therapy e.g. antidepressants and comparable to certain aforementioned active interventions for reducing symptoms of depression. Based on a preliminary review of the included studies, yoga may be recommended as an adjunctive treatment option for patients willing and able to learn and continue regular practice. Further RCT with higher quality methodology could be performed to clarify the role of yoga for treating depression including types of yoga, frequency of practice, comparisons to other therapies in larger studies and magnitude of effects. In addition trials assessing the long term effects, adherence and safety of yoga provide additional guidance for clinicians.

\section{Acknowledgements}

None.

\section{Conflict of Interests}

No conflict of interest.

\section{References}

1. (2018) National Health Interview Survey 2017. National Center for Complementary and Integrative Health, USA.

2. Prathikanti S, Rivera R, Cochran A, Tungol JG, Fayazmanesh N, et al. (2017) Treating major depression with yoga: A prospective, randomized, controlled pilot trial. PLoS One 12(3): e0173869.

3. Uebelacker L, Tremont G, Gillette L, Epstein Lubow G, Strong D, et al. (2017) Adjunctive yoga v. health education for persistent major depression: A randomized controlled trial. Psychol Med 47(12): 21302142.

4. Cramer H, Lauche R (2013) Langhorst J Yoga for depression: a systematic review and meta-analysis. Depress Anxiety 30(11): 1068-1083.

5. De Manincor M, Bensoussan A, Smith CA, Barr K, Schweickle M, et al. (2016) Individualized yoga for reducing depression and anxiety, and 
improving well-being: a randomized controlled trial. Depress Anxiety 33(9): 816-828.

6. Falsafi N (2016) A Randomized Controlled Trial of Mindfulness Versus Yoga: Effects on Depression and/or Anxiety in College Students. J Am Psychiatr Nurses Assoc 22(6): 483-497.
7. Cramer H, Anheyer D, Lauche R, Dobos G (2017) A systematic review of yoga for major depressive disorder. J Affect Disord 213: 70-77. 